\title{
Modeling of a Decision-making System for the Balancing of a Ship Based on Variable Weight
}

\author{
Yi Xianglie,Qiu Jinshui, \\ College of Naval Architecture and Power \\ Naval Univ. of Engineering \\ Wuhan, China, 430033
}

\author{
Liu Boyun \\ College of Naval Architecture and Power \\ Naval Univ. of Engineering \\ Wuhan, China, 430033
}

\begin{abstract}
- this paper presents a new method to build a decision-making model for the balancing of a Ship with variable weight. The main point is to show how to build a decision-making model for the balancing of a damaged ship more precisely than they do before, and can provide the decision maker to balance a damaged ship with a very direct and useful advice. Following the steps give in part six, you can easily build up the model of decision-making system for the balancing of a ship based on variable weight.
\end{abstract}

Keywords- Multi-objective, Variable Weight,Anti-sinking.

\section{INTRODUCTION}

At the same time or after limiting the spread of water of a damaged compartment or the closure of a damaged compartment, we should balance the ship appropriately, so that ship's status won't be deteriorated. Mainly there are three balancing approaches: moving water, unloading oil or water, excluding water, in order to build a decision-making model for the balancing of a ship, the three balancing approaches will be unified into a single model in this article. The decision-making system mainly includes two aspects: First, cabins which can be used for anti-sinking are listed in a certain order for users to choose; Second, to establish a model to search optimization balancing plan [1]

\section{MULTI-OBJECTIVE DECISION THEORY}

Contrapose to a damage state for a particular ship, the possible balancing plans, we need a theoretical approach for decision makers to choose an optimization balancing plan, this is a multi- objective decision-making. On the other hand, the anti-sinking of a ship involved in a lot of the survivability indexes. the anti-sinking goal is to make each of the survivability index reach a high level to the best of the status of a damaged ship; but the survivability indexes mutual restrain with each other, and some of those are even conflicting with each other, and can not simultaneously achieve the optimum. In this case, balancing plans must integrate in all aspects, so that we can get a optimal integrated index, this is a multi-objective decision making. Therefore, the anti-sink decision-making for balancing of a damaged ship is actually a multi-program multi-objective decision making problems.

To solve a multi-objective decision making problems, first of all, according to the requirements of the programe, transfer those highly overviewed of the rather vague objectives into more specific goals and then build the target sets, to construct a suitable model, and finally, do the compare analysis of a variety of feasible solution, using some optimization methods to sort the feasible solutions for decision makers to choose.

After a careful analysis d floating and stability datas of ships, and I have consulted many experts for advice, I decided to use horizontal angle, vertical angle, degree of initial stability, the minimum freeboard as four decisionmaking objectives, the program's effectiveness as a decision variable in this decision-making system, the effects of multiple programs may constitute the initial decision matrix. In the four decision-making objectives, the horizontal angle, vertical angle is a cost-based objective, and its value should be as small as possible; initial stability and minimum freeboard is high efficiency goal, the larger the better.

\section{STANDARDIZATION OF ANTI-SINKING INDICATORS}

According to the unsinkability's definition: after damaged and seawater flooded in, the ship's ability to float on the water surface without overturning, and continue to sail and combat. Therefore, the research of the unsinkability of a ship is to study the floating ability and stability of the ship after damage. So, after the ship has been flooded and damaged the threat is measured mainly through measurement of floating ability and initial stability, combining with the ship's hull arrangement and the weapons destruction effects to the ship [1]. Therefore, in this paper, the following four criteria are used as the comprehensive assessment indicators of a ship's unsinkability:

1. Index of initial stability $\mathrm{IH}$, transferred from the horizontal center height $\mathrm{h}$, and obtained by the following conversion formula:

$$
I H=\left\{\begin{array}{crl}
0 & h \leq R_{a} \\
1-\left(R_{b}-h\right)^{2} / R_{b}^{2} & R a<h \leq R_{b} \\
1 & R_{b}<h \leq R_{c} \\
\exp \left[-2 \cdot\left(h-R_{c}\right)^{2}\right] & h>R_{c}
\end{array}\right.
$$

Where:

h: damaged ship's initial stability.

$\mathrm{Ra}$ : according to the related specification, if the initial stability $\mathrm{h}$ is positive after the ship damage, $\mathrm{Ra}=0$. 
$\mathrm{Rb}: \mathrm{Rb}$ is equal to the cross stable center when the ship at the standard displacement.

Rc: $\mathrm{Rc}=1.1 \mathrm{Rb}$.

After conversion, the smaller the IH is, the threat of injury is higher.

2. Horizontal angle index. IR can be defined by the following formula:

$$
I R=1-\frac{\theta}{\theta_{a}}
$$

Where:

$\theta$ : horizontal angle of the damaged ship's heel (degrees).

$\theta \mathrm{a}$ : a ship's maximum horizontal angle which does not affect the use of combating operations, and generally 10 degrees.

3. Vertical angle index. IP can be defined by the following formula.

$$
I P=1-\frac{\varphi}{\varphi_{a}}
$$

Where: (degrees).

$\varphi$ : The ship's damaged lengthways incline angle

$\varphi_{a}$ : The ship's maximum lengthways incline angle which does not affect the use of combating operations, and generally 3 degrees.

4. The minimum freeboard index IG. And can be expressed as:

$$
I G=\frac{1}{1+\exp \left[2-\frac{4(G X-S G)}{0.45 L G-S G}\right]}
$$

Where:

GX: The height of the minimum freeboard of the damaged ship.

LG: The height of the freeboard amidships when the ship at full load displacement.

SG: constraint value which the related specification allowed, generally $\mathrm{SG}=0.6$.

\section{Anti-Sinking EFFECT EVALUATION INDEX}

Set the current ships status as V0, the current initial stability as h0, angle of horizontal angle as $\theta 0$, vertical angle as $\psi 0$, the minimum freeboard height Fmin0; set the ship status after anti-sinking as V1, the initial stability as h1, horizontal angle as $\theta 1$, vertical angle as $\psi 1$, the minimum freeboard height Fmin1.

1. Restoration of Stability Index: $E_{h}=\frac{h_{1}-h_{0}}{h_{a}-h_{b}} * \frac{1}{2}+\frac{1}{2}$

2. The effect of horizontal angle recovery index:

$$
E_{\text {Heel }}=\frac{\theta_{1}-\theta_{0}}{\theta_{\mathrm{a}}-\theta_{\mathrm{b}}} * \frac{1}{2}+\frac{1}{2}
$$

3. Effect of vertical angle recovery index:

$$
E_{\text {Trim }}=\frac{\psi_{1}-\psi_{0}}{\psi_{\mathrm{a}}-\psi_{\mathrm{b}}} * \frac{1}{2}+\frac{1}{2}
$$

4. The minimum freeboard recovery effect index:

$$
E_{F \min }=\frac{F \min _{1}-F \min _{0}}{F \min _{\mathrm{a}}-F \min _{\mathrm{b}}} * \frac{1}{2}+\frac{1}{2}
$$

\section{Variable Weight Decision-Making Method}

Ship's Anti-sinking is a complex decision-making project, in decision-making, we should take into account a number of indexes of the balance target. In the current antisinking decision-making, it only takes into account the relative importance of each indexes in decision-making (the weight is constant), and neglect the state preference degree of the balance adjustment, this "constant versus great changes "approach has a certain one-sidedness. For example: commonly, there are four factors in the anti-sinking plan: initial stability, horizontal angle, vertical angle and minimum freeboard height, if considered as of same importance in decision-making, the decision target is $\mathrm{V}=0.25 \mathrm{x}_{1}+0.25 \mathrm{x}_{2}+0.25 \mathrm{x}_{3}+0.25 \mathrm{x}_{4} \quad\left(\mathrm{x}_{1}\right.$ is the initial stability index, $\mathrm{x}_{2}$ is the horizontal angle index, $\mathrm{x}_{3}$ is the vertical angle index, $x_{4}$ the minimum freeboard index), in state $\mathrm{A}, \mathrm{x}_{1}=\mathrm{x}_{2}=\mathrm{x}_{3}=\mathrm{x}_{4}=0.5$ is reasonable, while in state $B$, where $\mathrm{x}_{1}=0.1, \mathrm{x}_{2}=0.9, \mathrm{x}_{3}=0.5, \mathrm{x}_{4}=0.5$, obviously does not match with the actual. As anti-sinking is a dynamic process, the method in which the states change while the weights keep constant is clearly unreasonable, and in the anti-sinking decision-making, to improve the the most dangerous targets is of foremost importance.

In order to solve these problems, combined with variable weight theory, in the establishment of state indices and anti-sinking effect of index based on the combination KEPNER-TREGOE decision making, the establishment of a multi-level multi-objective model for integrated decisionmaking variable weights, so that a comprehensive antisinking system decision-making more scientific and reasonable.

1. Variable Weight Theory

Factor space theory is a new theory proposed and studied by Professor Li Hong-xing and Wang Pei-Chuang. Among them, the comprehensive principle of variable weight is an important principle in the modeling process, it reflects the every elements state of balance in the integrated decision-making. Like the example given above, the costant weight method is inappropriate in decision-making in many cases. In the normal state, the costant weight method integrated model can be shown as[2]:

$$
V_{0}=\sum_{j=1}^{m} w_{j}^{(0)} x_{j}
$$

And should be amended to changing weights method:

$$
V=\sum_{j=1}^{m} w_{j}\left(x_{1}, \cdots x_{m}, w_{1}^{(0)}, \cdots w_{m}^{(0)}\right) x_{j}
$$

changing weights method's weights changing formula: 


$$
w_{j}\left(x_{1}, \cdots x_{m}\right)=w_{j}^{(0)} x_{j} / \sum_{j=1}^{m} w_{j}^{(0)} x_{j}
$$

Brief introduction of a few important definitions of variable weight theory.

1) weight vector regularly defined as $W_{0}=\left(w_{1}, \cdots, w_{m}\right) \in(0,1]^{m} \quad$ (often referred to the constant), and $\sum_{j=1}^{m} w_{j}=1$.

2) for a given map $W:[0,1]^{m} \rightarrow(0,1]^{m}$, call it the variable weight vector $W(X)=\left(w_{1}(X), \cdots w_{m}(X)\right)$, if it can measure up the following conditions:

w.1) normalization: $\sum_{j=1}^{m} w_{j}\left(x_{1}, \cdots x_{m}\right)=1$;

w.2) continuity for each element:

$\forall j(1 \leq j \leq m), w_{j}\left(x_{1}, \cdots x_{m}\right)$ continuous with every variable $x_{k}(k=1, \cdots m)$;

w.3) incentive: $w_{j}\left(x_{1}, \cdots x_{m}\right)$ on a single increase with $x_{j}$, or

w.3 ') penalty: $w_{j}\left(x_{1}, \cdots x_{m}\right)$ on the single drop with $x_{j}$. and $\mathrm{W}$ satisfy w.1),

if satisfy w.1), w.2),w.3), called W-type variable weight vector for the punishment variable weight vector; if $\mathrm{W}$ satisfy w.1), w. 2) and w.3 '), called W for the incentive variable weight vector.

3) Given $\operatorname{map} S:[0,1]^{m} \mapsto(0,1]^{m}$, $X \rightarrow S(X) \underline{=}\left(S_{1}(X), \cdots S_{m}(X)\right)$ if the conditions:

s.1) $S_{j}\left(\sigma_{i j}(X)\right)=S_{j}(X)$, here:

$\sigma_{i j}\left(x_{1}, \cdots, x_{i}, \cdots x_{j}, \cdots, x_{m}\right)=\left(x_{1}, \cdots, x_{j}, \cdots, x_{i}, \cdots, x_{m}\right)$;

s.2) $x_{i} \geq x_{j} \Rightarrow S_{i}(X) \leq S_{j}(X)$;

s.3) $S_{j}\left(x_{1}, \cdots, x_{m}\right)$ for every variable continuous;

s.4) for any constant power $W_{0}=\left(w_{1}, \cdots, w_{m}\right)$, set

$$
W(X)=\frac{W_{0} \bullet S(X)}{\sum_{j=1}^{m}\left(w_{j} S_{j}(X)\right)}
$$

If it can satisfy the conditions of Definition 2, then we say $\mathrm{S}$ is the state variable weights vector; then, if satisfied w.1), w.2), w.3), called $S$ as the incentive state variable weight vector; If you meet the w. 1), w.2), w.3 '), S will be called a penalty-type state variable weights vector.

In the balancing processof a ship, in the basis for our decision-making, we always set the most dangerous targets as first choice for the decision, that the smaller the state index is, the greater the weight of it is, it is a punitive state variable weights vector.

\section{VARiable Weight Integrated Decision Model}

1) Assess the risk of each status index of the damaged ship; calculate the weight of each index by the listed formula.

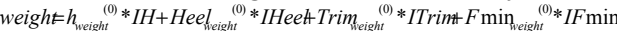

$$
\begin{aligned}
& h_{\text {weight }}=h_{\text {weight }}{ }^{(0)} * I H / \text { weight } \\
& \text { Heel }_{\text {weight }}=\text { Heel }_{\text {weight }}{ }^{(0)} * \text { IHeel } / \text { weight } \\
& \text { Trim }_{\text {weight }}=\operatorname{Trim}_{\text {weight }}{ }^{(0)} * \text { ITrim } / \text { weight } \\
& F \min _{\text {weight }}=F \min _{\text {weight }}{ }^{(0)} * I F \min / \text { weight }
\end{aligned}
$$

Where the $h_{\text {weight }}{ }^{(0)}$ Heel $_{\text {weight }}{ }^{(0)} \operatorname{Trim}_{\text {weight }}{ }^{(0)} F \mathrm{~min}_{\text {weight }}{ }^{(0)}$ is the weight of each index of the damaged ship, and can be determined by the analysis their importance given by related experts.

2) Initialize the balance program. As follows: assume the number of compartments of the ship which are not damaged and can import oil and water as $m$, the number of compartments of the ship which are not damaged and can export oil and water as $n$. so there are $m$ kinds of solutions of ballast, $\mathrm{n}$ kinds of solutions of uninstalled, all the shift should be done according to the nature of the tank, where only the compartments with the same nature can lead the shift (such as oil - oil , fresh water - fresh water). Here, in order to reduce free surface effect, we assume that each time, the unloading is emptied, the ballast is full.

3) Assess the effect of each program. Specific methods are as follows:

According to the ship status after the application of the program, compute the effect index of each state recovery $E_{h}, E_{\text {Heel }}, E_{\text {Trim }}, E_{F \text { min }}$, and then calculated the total program effect index.

$$
E=E_{h} * h_{\text {weight }}+E_{\text {Heel }} * \text { Heel }_{\text {veight }}+E_{\text {Trim }} * \text { Trim }_{\text {weight }}+E_{F \min } * F \text { min }_{\text {veighi }}
$$

4) Sort the effects of each program, set the best solution as the first choice. According to the previous Modeling process, the program can improve the ship's survivability when the overall effect index is greater than 0.5 , the other hand, if less than 0.5 will make the situation worse, the program is not feasible.

5) After the assessment of the first anti-sinking program's effect, if the program can satisfy the requirements, stop the program, else set the results of the first program as the initial state, and re-evaluate the status of ship after the application of the anti-sinking program.

6) Re-initialize the balancing program. Do as the second step, but not included in the compartments have been used before.

7) Repeat 3. 4. 5, until you meet all the requirements.

8) evaluate the possibility of realizing the program, and make the final decision to choose the best ant-sinking program[3][4].

Specific flow chart shown in Fig.1. 


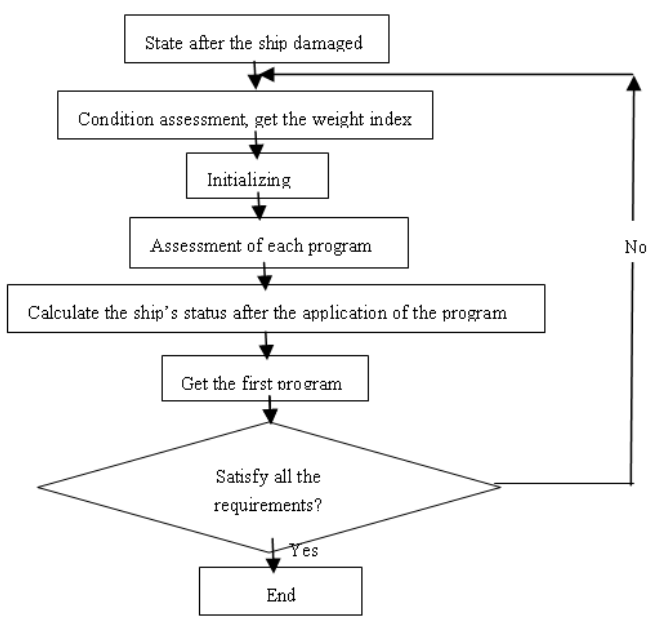

Figure 1. Flow chart of the modeling process

\section{SUMMARIES}

Now, we can draw some conclusions about the process of the model building of the decision-making system for the balancing of a damaged ship. This kind of modeling is obviously more precise and efficient than those before, if our decision-maker adopt the decision-making system built before; there are obviously some mistakes or even worse, coursing the damaged ship's status getting worse. But, this kind of modeling described in this paper can do a better job, and will become a good assistant for the decision-maker of the balancing for a damaged ship.

\section{REFERENCES}

[1] PU Jin-yun; JIN Tao; QIU Jin-shui. Warship's Survivability. Press Defense Industry. 2008, PP: 256-257.

[2] ZHANG Yu-zhuo; LI Yu-cheng; LI Hong-xing. The Application of Variable Weight Algorithm In The Fussy Illation. Fussy System And Mathematics. 2006, PP: 66-72.

[3] NWC-TN-4565-3-71:Voll and 2. MAGIC Computer Simulation: Vol1. User Manual: Vol2. Analyst Manual[S]. Armament Systems Inc. and Propulsion Development Department, 1971.

[4] Cudey Donald E; James B Flint. Target Description Computer Programs, Techniques for Developing Input Data[R]. AD379263L, 1967, PP: 3-13. 